This is the peer reviewed version of the following article "Aerodynamic design optimization of wind turbine rotors under geometric uncertainty", by M.S. Campobasso, E. Minisci, M. Caboni, Wind Energy, 2016, which has been published in final form at https://doi.org/10.1002/we.1820. This article may be used for non-commercial purposes in accordance with Wiley Terms and Conditions for Use of Self-Archived Versions.

Aerodynamic design optimization of wind turbine rotors under geometric uncertainty

M. Sergio Campobasso, E. Minisci, M. Caboni

Wind Energy, Vol. 19, no. 1, 2016, pp. 51-66.

First published: 14 November 2014 


\section{AERODYNAMIC DESIGN OPTIMIZATION OF WIND TURBINE ROTORS UNDER GEOMETRIC UNCERTAINTY}

\author{
M. Sergio Campobasso \\ Lancaster University \\ Department of Engineering \\ Engineering Building \\ Lancaster LA1 4YR, United Kingdom \\ m.s.campobasso@lancaster.ac.uk
}

\author{
Edmondo Minisci \\ University of Strathclyde \\ Department of Mechanical and \\ Aerospace Engineering \\ 75 Montrose Street \\ Glasgow, G1 1XJ, United Kingdom \\ edmondo.minisci@strath.ac.uk
}

November 16, 2014

\footnotetext{
*Address all correspondence to this author.
} 


\begin{abstract}
Presented is a robust optimization strategy for the aerodynamic design of horizontal axis wind turbine rotors including the variability of the annual energy production due to the uncertainty of the blade geometry caused by manufacturing and assembly errors. The energy production of a rotor designed with the proposed robust optimization approach features lower sensitivity to stochastic geometry errors with respect to that of a rotor designed with the conventional deterministic optimization approach that ignores these errors. The geometry uncertainty is represented by normal distributions of the blade pitch angle, and the twist angle and chord of the airfoils. The aerodynamic module is a blade-element momentum theory code. Both Monte Carlo sampling and the univariate reduced quadrature technique, a novel deterministic uncertainty analysis method, are used for uncertainty propagation. The performance of the two approaches is assessed in terms of accuracy and computational speed. A two-stage multi-objective evolutionbased optimization strategy is used. Results highlight that, for the considered turbine type, the sensitivity of the annual energy production to rotor geometry errors can be reduced by reducing the rotational speed and increasing the blade loading. The primary objective of the paper is to highlight how to incorporate an efficient and accurate uncertainty propagation strategy in wind turbine design. The formulation of the considered design problem does not include all the engineering constraints adopted in real turbine design, but the proposed probabilistic design strategy is fairly independent of the problem definition and can be easily extended to turbine design systems of any complexity.
\end{abstract}

KEYWORDS: wind turbine rotor design; stochastic geometry errors; manufacturing tolerances; probabilistic design optimization. 


\title{
Nomenclature
}

\author{
$A E P \quad$ Annual energy production. \\ $B M \quad$ Root bending moment. \\ $D E \quad$ Differential evolution. \\ EA Evolutionary algorithm. \\ $H A W T$ Horizontal axis wind turbine. \\ $I D E A \quad$ Inflationary differential evolution algorithm. \\ $M C \quad$ Monte Carlo. \\ MOPED Multi-objective Parzen-based estimation of distribution. \\ $P D F \quad$ Probability density function. \\ $N_{d} \quad$ Number of design variables. \\ $R \quad$ Tip radius. \\ $S S C \quad$ Stochastic simplex collocation. \\ TSR Tips speed ratio. \\ $U \quad$ Freestream wind velocity. \\ $U_{\text {rel }} \quad$ Relative wind velocity. \\ $U R Q \quad$ Univariate reduced quadrature. \\ $n \quad$ Number of uncertain design variables. \\ $r \quad$ Radius along the blade. \\ $\mathbf{x} \quad$ Array of design variables. \\ $\alpha \quad$ Angle of attack. \\ $\theta_{p} \quad$ Section pitch angle. \\ $\theta_{p, 0} \quad$ Blade pitch angle. \\ $\theta_{T} \quad$ Blade twist angle. \\ $\mu \quad$ Expectation. \\ $\sigma \quad$ Standard deviation. \\ $\sigma^{2} \quad$ Variance. \\ $\phi \quad$ Angle of relative wind. \\ $\Omega \quad$ Rotational speed.
}




\section{Introduction}

The growing availability of large computational resources and progress of design optimization technologies offer the means to automate significant portions of product design. In the past few years, several studies on the use of diverse optimization techniques for the preliminary design of horizontal axis wind turbines (HAWTs) have appeared. Some of these applications have focused on the optimization of existing blades by means of local search approaches [1, 2, 3, 4], utilizing low- to medium-fidelity models. Global multi-objective evolution-based search methods have also been used, often to optimize HAWT conceptual designs, and investigate the choice of fundamental HAWT design parameters, such as its rotor diameter, on the economy of whole wind farms [5].

One way in which modern HAWT design could be further improved is by accounting for the effects of environmental, operational and engineering uncertainty throughout the design process. The use of uncertainty management and quantification tools increases computational costs, and this motivates the efforts to develop new approaches allowing these technologies to be efficiently integrated in HAWT design. Accounting for the impact of uncertainty in HAWT design requires the use of numerical methods which can reliably propagate uncertainty throughout the design system without keeping HAWT design computationally unaffordable. The conceptually simplest way to propagate uncertainty through an analysis system is to sample the design space using Monte Carlo (MC) methods [6]. Unfortunately, MC methods are computationally expensive, requiring a large number of function evaluations to converge. Therefore, researchers have been developing alternative, computationally cheaper approaches to uncertainty propagation. The main difficulty is to reduce computational costs with respect to $\mathrm{MC}$ methods while maintaining an acceptable accuracy of the probabilistic parameters of the output values. The techniques that have been proposed to accomplish these two conflicting requirements range from the Taylor-based method of moments [7, 8] to quadrature methods [9] and polynomial chaos expansion [10]. Among the proposed alternatives, an appealing one is the Univariate Reduced Quadrature (URQ) approach [11], which has been successfully used for the robust shape optimization of a transonic airfoil by means of a local gradient based search. The use of this deterministic sampling technique in robust design optimization based on global search methods is appealing and promising, but so far the URQ uncertainty propagation technique has not been used in global design optimization. As shown below, the use of URQ in the context of robust design optimization of HAWT rotors based on global search method is one of the novel elements of this paper.

This paper focuses on the development and the demonstration of a general methodology to incorporate uncertainty in HAWT design. To the best of the authors' knowledge, this issue has so far received little attention despite the significant implications it may have on HAWT design, turbine energy production and, ultimately, cost of energy. One of the sources of engineering uncertainty is the effect of blade geometry errors caused by finite manufacturing and assembly tolerances on the power and, for a selected site, the energy production of the turbine. The problems associated with deviations of the actual blade geometry from its nominal shape is mentioned in [12], and a few preliminary investigations of this matter are reported in [13]. Petrone et al. [14] have studied the impact of blade twist errors due to finite manufacturing tolerances, and also wind speed, turbulence intensity and wind direction variability, and blade surface roughness variations caused by insect contamination on the mean power coefficient and acoustic emissions of a stall-regulated HAWT. These authors have used Latin Hypercube Sampling and the Stochastic Simplex Collocation (SSC) method to propagate uncertainties throughout the multi-disciplinary analysis system. Petrone et al. [15] have also optimized the blade geometry of a stall-regulated rotor for maximum mean power coefficient and minimum acoustic emissions considering the uncertainty on laminar-to-turbulent transition caused by uncertain blade surface roughness levels. Uncertainty has been propagated 
using the SSC method, and the optimization has been carried out using a multi-objective genetic algorithm.

This paper presents a new computationally very fast method to account for uncertainty in HAWT design optimization, resulting in a computationally affordable HAWT probabilistic design optimization framework. The considered sample application is the aerodynamic design of a variablespeed fixed-pitch stall-regulated HAWT rotor, subject to the uncertainty associated with stochastic blade geometry errors due to finite manufacturing and assembly tolerances (in the remainder of the paper, 'rotor' denotes the turbine blades, and 'rotor geometry' denotes the blade geometry and their pitch angle.) A detailed investigation into the effects of the stochastic variations of the rotor geometry on the level and variability of the turbine power is presented. Use of the probabilistic design optimization framework yields a robust aerodynamic design, namely a rotor, the aerodynamic performance of which has minimal sensitivity to blade geometry errors.

The aerodynamic module of the design system is a blade-element momentum theory code. Uncertainty is propagated using the rapid URQ technique, and an evolution-based optimization approach is used. More specifically, the adopted evolution-based global search method is a twostage optimization process: the first stage is based on a multi-objective Estimation of Distribution algorithm [16, 17], which has been shown to have very good exploratory capabilities; the second stage uses a differential evolution-based approach [18], which has good exploitation capabilities. It should be noted that, even when only one objective function is considered, robust design optimization problems can be viewed as multi-objective optimization problems. This is because one has to optimize both the expectation and the standard deviation of the objective function, which, in the present study, is the HAWT energy production, as shown below. Hence, one of the main reasons for using evolution-based optimization in this study was that this technology can easily handle multi-objective problems. Another reason for this choice is the global exploration character of evolutionary methods, which offers the possibility of considering fairly radical new designs.

Due to some modeling limitations of the adopted aerodynamic analysis tools (see below), the optimal design solutions presented in this study may require further verification. The main objective of the robust turbine design optimization exercise presented herein, however, is to explore the potential of robust design optimization for improving general multi-disciplinary HAWT design technologies [19], rather than proposing new design solutions for a particular turbine type. The reason for selecting a variable-speed fixed-pitch stall-regulated turbine is merely that this work stemmed from research collaborations of the authors with manufacturers of relatively small HAWTs, and the selected turbine layout is often adopted for small wind turbines.

The paper first describes the optimization approach. This is followed by the definition of the probabilistic design optimization problem. The following section presents a comparative numerical study of the MC and URQ approaches to uncertainty propagation aimed at assessing their accuracy and computational cost in view of their use within the global evolution-based optimizers used in this study. The result section reports the findings of two slightly different variants of the same general robust optimization problem. It also provides a comparative analysis of two optimized rotor geometries, one obtained by neglecting uncertainty (deterministic optimization), and the other obtained by including the aforementioned geometry uncertainty (robust optimization). The main conclusions of the study are summarized in the closing section.

\section{Optimizers}

Evolutionary Algorithms (EAs) solve optimization problems by making a generation of individuals evolve subject to selection and search operators. In this study, an individual denotes a HAWT rotor 
configuration, defined by the geometry of the its blades and its range of rotational speeds. This iterative process eventually leads to a population containing the fittest possible individuals (best rotor configuration designs), or individuals who are significantly fitter than those of the starting population. The role of the selection operators is to identify the fittest or most promising individuals of the current population, whereas search operators such as crossover and mutation attempt to generate better offspring starting from suitably selected individuals of the current generation. Each individual is defined by genes, which correspond to design variables in design optimization. The solution of the optimization problems reported in this study is based on a two-stage approach using the Multi-Objective Parzen-based Estimation of Distribution (MOPED) [16] and the Inflationary Differential Evolution Algorithm (IDEA) [18].

MOPED belongs to a subset of EAs and was developed to circumvent certain algorithmic problems of conventional EAs, which can be ineffective when the problem at hand features a high level of interaction among the design variables.

This is mainly due to the fact that the recombination operators are likely to disrupt promising sub-structures that may lead to optimal solutions. Additionally, the use of the crossover and mutation operators may result in slow convergence to the solution of the optimization; that is, it may require a large number of generations to obtain very fit individuals. MOPED was developed to circumvent shortfalls of this kind. Its use of statistical tools enables it to preserve promising substructures associated with variable interaction from one generation to another (automatic linkage learning). Such statistical tools also replace the crossover and mutation operators of conventional EAs, and they allow a faster convergence of MOPED with respect to the latter class of optimizers. Starting from the individuals of the current population, MOPED builds an approximate probabilistic model of the search space. The role of the crossover and mutation operators is replaced by sampling of this probabilistic model. There exist similar other evolutionary methods that use the aforementioned strategy, and they are called Estimation of Distribution Algorithms [20]. MOPED is a multi-objective optimizer for continuous problems that belongs to this class of algorithms and uses the Parzen method [21] to build a probabilistic representation of Pareto solutions, and can handle multivariate dependencies of the variables $[16,17]$. MOPED implements the general layout and the selection techniques of the Non-dominated Sorting Genetic Algorithm II (NSGA-II) [22], but traditional crossover and mutation search approaches of NSGA-II are replaced by sampling of the Parzen model. NSGA-II was chosen as the base for MOPED mainly due to its simplicity, and also for the excellent results obtained for many diverse optimization problems [23, 24].

The Parzen method utilizes a non-parametric approach to kernel density estimation, and results in an estimator that converges asymptotically to the true Probability Density Function (PDF) over the whole design space. Additionally, when the true PDF is uniformly continuous, the Parzen estimator can also be made uniformly consistent. The Parzen method allocates $N_{\text {ind }}$ identical kernels (where $N_{\text {ind }}$ is the number of individuals of the current population), each centered on a different element of the sample. A probabilistic model of the promising search space portion is built on the basis of the statistical data provided by the $N_{\text {ind }}$ individuals through their kernels, and $\tau_{E} N_{\text {ind }}$ new individuals $\left(\tau_{E} \geq 1\right)$ are sampled. The variance of each kernel depends on $(i)$ the location of the individuals in the search space and $(i i)$ the fitness value of these individuals, and its construction leads to values that favor sampling in the neighborhood of the most promising solutions.

The features of MOPED often prevent the true Pareto front from being achieved, particularly when the front is broad and the individuals of the population are spread over different areas, which are far apart from each other in the feasible space. This circumstance has prompted coupling MOPED with another EA, which has better convergence properties. To this aim, the Inflationary Differential Evolution Algorithm has been selected. IDEA was first developed for the design opti- 
mization of interplanetary trajectories, and it is an improved variant of the differential evolution (DE) algorithms [18]. The IDEA algorithm is based on a synergic hybridization of a standard DE algorithm and the strategy behind the monotonic basin hopping [25]. The resulting algorithm was shown to outperform both standard DE optimizers and the monotonic basin hopping algorithm in the solution of challenging space trajectory design problems, featuring a multiple funnel-like structure. In this paper, a modified version of IDEA has been used to move the individuals of the approximate Pareto front obtained with MOPED closer to the true front.

The main features of the original IDEA algorithm are reported in [18]. The IDEA algorithm works as follows: a DE process is performed several times and each process is stopped when the population contracts below a predefined threshold. At the end of each DE step, a local search is performed in order to get closer to the local optimum. In the case of non-trivial functions, where there is a high likelihood of converging to local optima, the combined DE/local search is usually iterated several times, performing either a local or a global restart on the basis of a predefined scheduling.

The design optimization presented in this study is constrained. Therefore, the DE step has been modified so that the fitness assessment of the individuals during the DE process also takes into account the constraints. The constraint handling technique used herein is one of the approaches that can be adopted in evolutionary computing, and is similar to the approach used by MOPED. In the unconstrained DE algorithm [26], and also in the unconstrained IDEA algorithm [18], each parent solution is compared with its offspring, and the solution with a better value of the objective function is passed to the next generation. In the constrained case, on the other hand, when parents and offspring are compared, the solutions are first evaluated in terms of constraint compatibility $c p$. Its definition is:

$$
c p(\boldsymbol{x})=\sum_{j=1}^{m} s_{j}(\boldsymbol{x})
$$

where $\mathbf{x}$ is the array of design variables, $m$ is the number of constraints, and the constraint factor $s_{j}$ is:

$$
s_{j}(\boldsymbol{x})=\max \left\{g_{j}(\boldsymbol{x}), 0\right\}
$$

The constraint factor equals 0 when the constraint $\left(g_{j}(\boldsymbol{x}) \leq 0\right)$ is satisfied and is strictly positive when the constraint is violated. The solution with the better values of $c p$ is then passed to the next generation. When the $c p$ values of parent and offspring are the same, the selection is performed on the basis of the objective function.

In the current implementation, MOPED and IDEA are used sequentially. When MOPED has reached a given number of generations, its final population represents a first and good approximation to the sought Pareto front. Then, clustered sub-populations of such a population are used as initial solutions of the single-objective constraint IDEA optimizer. this algorithm 'pushes' the individuals of a sub-population of the MOPED front towards a better local approximation of the sought Pareto front. The resulting two-stage optimizer blends the exploratory capabilities of MOPED (global exploration) and the favorable convergence characteristics of IDEA (exploitation of local information).

\section{Problem set-up}

\section{1 design problem definition}

In all HAWT rotor design optimizations reported below, the objective function to be maximized is the annual energy production (AEP) of a three-blade turbine, and this output is taken to be 
the yearly amount of mechanical energy at the turbine shaft. The yearly frequency distribution of the freestream wind velocity $U$ is represented by a Weibull distribution with scale parameter of $7 \mathrm{~m} / \mathrm{s}$ and shape parameter of 2 , resulting in a yearly average speed of $6.2 \mathrm{~m} / \mathrm{s}$. The turbine is regulated by varying its rotational speed $\Omega$ until its rated speed, and stall-regulated thereafter. The design specifications are that the blades feature a single airfoil geometry, namely the NACA4413 airfoil, the root and tip radii of the blades be fixed to $1.3 \mathrm{~m}$ and $6.3 \mathrm{~m}$, respectively. and the rated wind speed vary between 10 and $12 \mathrm{~m} / \mathrm{s}$. The nominal blade shape is parametrized by means of one design parameter defining the blade pitch angle $\theta_{p, 0}$, six design parameters defining the radial distribution of the twist angle $\theta_{T}$, i.e.the rotation of the considered blade section with respect to $\theta_{p, 0}$, and six design parameters defining the radial distribution of the chord $c$, The angles $\theta_{p, 0}$ and $\theta_{T}$ are reported in Fig. 1, along with the overall sectional pitch $\theta_{p}=\theta_{T}+\theta_{p, 0}$. Figure 1 also reports the angular speed vector $\omega$, the freestram wind velocity $U$, and the relative wind speed velocity vector $U_{r e l}$ observed by the considered section. The vector $U_{r e l}$ is inclined by an angle $\phi$ on the rotor plane, and the local angle of attack is therefore $\alpha=\phi-\theta_{p}$.

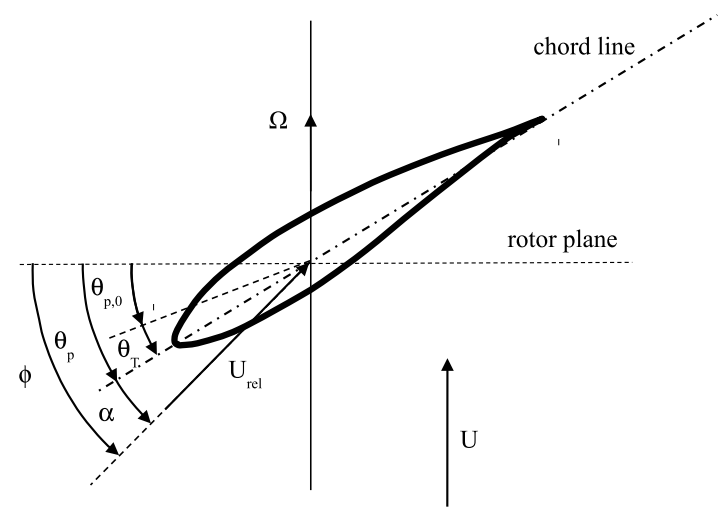

Figure 1: Geometric and aerodynamic parameters of a generic blade section.

The rotational speed $\Omega$ associated with each wind speed is also a design variable. Since seven wind speeds have been considered, given by $U_{i}=5+i, \quad i=1,7$, there are seven additional design variables, which are the seven rotational speeds $\Omega_{i}$ associated with the seven wind speeds $U_{i}$. The overall number of design parameters is thus $N_{d}=20$. The formulation of the design optimization problem could be slightly simplified by not including the blade pitch in the set of independent variables, but rather taking it as the twist of a section at a reference blade height. Nevertheless it has been preferred to keep the blade pitch as an independent design variable to test this approach in view of using the developed design system also for HAWTs adopting pitch control for power regulation. One could also replace the seven design variables associated with the rotational speeds with only the tip speed ratio (TSR). Without active constraints, the two approaches lead similar results, corresponding to fairly constant optimal tip speed ratio. Here the choice of taking $\Omega$ to be a general function of $U$ has been made for further verifying the implementation of the optimization system in view of its use with an aeroacoustic constraint, which can become active even before the rated wind speed, thus preventing a constant optimal TSR until rated wind speed. An additional reason for making and testing this modeling choice has been its forthcoming use in the design optimization of modern multi-megawatt HAWTs. In this machines the transition from the constant optimal TSR region before the rated wind speed to the constant power region is smooth and occurs over a sizable wind speed interval where TSR can take significantly sub-optimal values well before the rated wind speed. The adopted modeling choice will also enable accounting for such variability 
in HAWT design optimization.

The design variables $x_{1}$ to $x_{6}$ are the values of the chord $c$ at the radial positions

$\left(\begin{array}{llllll}r_{1} & r_{2} & r_{3} & r_{4} & r_{5} & r_{6}\end{array}\right)=\left(\begin{array}{llllll}1.3 & 2 & 3 & 4 & 5 & 6.3\end{array}\right) \mathrm{m}$, and the design variables $x_{7}$ to $x_{12}$ are the values of the twist angles $\theta_{T}$ at the same six radial positions. The shape of the blade is reconstructed by using the MATLAB ${ }^{\circledR}$ shape-preserving piecewise cubic interpolation function pchip over the six radial stations. The variable $x_{13}$ is the blade pitch angle $\theta_{p, 0}$, and the variables $x_{14}$ to $x_{20}$ are the seven values of $\Omega$ associated with the seven considered values of $U$. The bounds of all design variables are given in Table 1.

\begin{tabular}{|c|c|c|}
\hline$x_{1}$ & $\in[0.10,0.90] m$ & $c\left(r_{1}\right)$ \\
\hline$x_{2}$ & $\in[0.10,0.90] m$ & $c\left(r_{2}\right)$ \\
\hline$x_{3}$ & $\in[0.10,0.90] m$ & $c\left(r_{3}\right)$ \\
\hline$x_{4}$ & $\in[0.10,0.60] m$ & $c\left(r_{4}\right)$ \\
\hline$x_{5}$ & $\in[0.10,0.50] m$ & $c\left(r_{5}\right)$ \\
\hline$x_{6}$ & $\in[0.10,0.40] m$ & $c\left(r_{6}\right)$ \\
\hline$x_{7}$ & $\in[0,50] d e g$ & $\theta_{T}\left(r_{1}\right)$ \\
\hline$x_{8}$ & $\in[0,50] d e g$ & $\theta_{T}\left(r_{2}\right)$ \\
\hline$x_{9}$ & $\in[0,40] d e g$ & $\theta_{T}\left(r_{3}\right)$ \\
\hline$x_{10}$ & $\in[0,20] d e g$ & $\theta_{T}\left(r_{4}\right)$ \\
\hline$x_{11}$ & $\in[0,10] d e g$ & $\theta_{T}\left(r_{5}\right)$ \\
\hline$x_{12}$ & $\in[0,10] d e g$ & $\theta_{T}\left(r_{6}\right)$ \\
\hline$x_{13}$ & $\in[-10,10] d e g$ & $\theta_{p, 0}$ \\
\hline$x_{14-20}$ & $\in[50,150] r p m$ & $\Omega_{i}\left(U_{i}\right), i=1,7$ \\
\hline
\end{tabular}

Table 1: Range of design variables.

The 13 independent parameters $\left(x_{i}, i=1,13\right)$ defining the blade geometry are assumed to be affected by non-correlated normally distributed uncertainty. The joint Gaussian distribution of these 13 parameters is centered at the nominal values of the corresponding design variable, and has a standard deviations of $1 \mathrm{~cm}$ for lengths and about 2.5 degrees for angles. The order of magnitude of these standard deviations is representative of the geometry errors observed for the blade size considered in this study. Since the optimizers use a nondimensionalized search space, with the input variables varying in the interval $[0,1]$, the vector of nondimensional standard deviations of each blade, obtained by dividing each standard deviation by the dimensional range of the corresponding variable, is $\sigma_{1}=\left[\begin{array}{ll}0.0125 & 0.01250 .01250 .020 .025\end{array}\right.$

0.03330 .050 .050 .050 .10 .20 .20 .1 . The stochastic blade shape geometry errors may induce some uncertainty in the dependence of the power coefficient on TSR, and, hence, also on the optimal values of the latter parameter, which determine the optimal rotational speeds for each wind speed. The impact of the variability of $\Omega$ due to the blade geometry errors on the AEP variability, however, is likely to depend of the adopted control strategy [27]. Using maximum power tracking, for example, the rotational speed is set by choosing in real time the value that maximizes the turbine power, the turbine works at its true optimal rotational speed all times, and variations of $\Omega$ with respect to its nominally optimal value contribute fairly little to the power variability. Conversely, the variations of the optimal $\Omega$ caused by geometry errors may contribute more to such power variability when the machine model is used directly by the controller, like in the cases in which the controller attempts to track the nominally optimal TSR or the nominally optimal aerodynamic torque. This is because the rotor may be forced to work at a speed significantly different from its actual optimum. In practice, 
however, rotational speed controllers are affected by additional and expectedly larger uncertainty sources, such as low accuracy by which required input data like wind speed can be measured [27], and also high spatial and temporal wind variability resulting in significant deviations from the often overly simple machine model used by the controller. In this study, only the uncertainty on the energy production caused by the dependence of blade aerodynamics on the probabilistic blade geometry errors is analyzed. The analysis of the uncertainty of turbine controllers is beyond the scope of the article. Therefore, rather than considering in an incomplete manner the uncertainty on affecting $\Omega$, it has been preferred to treat the rotational speeds as deterministic variables. The incorporation of all uncertainty sources affecting the turbine speed control in a probabilistic multidisciplinary HAWT design system will be treated in future extensions of this work.

The power curve of the turbines analyzed in the design optimization have been computed using WINSTRIP, an in-house FORTRAN code implementing the blade-element momentum (BEM) theory [28]. Theoretically BEM codes have a relatively narrow range of applicability, but several semi-empirical correction models enable their use for a wider range of operating conditions. The corrections implemented in WINSTRIP include: a) Prandtl's tip and hub loss corrections [28], $b$ ) Glauert's correction [28] improved by Buhl [29] to account for axial induction factors exceeding the maximum theoretical limit of 1/2, and c) Snel's [30] and the AERODAS [31] corrections to account for the rotational effect known as Himmelskamp or centrifugal pumping effect, occurring in the presence of large areas of stalled flow. WINSTRIP has been validated against the experimental data of the NREL 2-blade UAE phase-VI test turbine [32], and a good agreement has been observed. In the present work, the airfoil lift and drag data required by the BEM code have been computed using the Massachusetts Institute of Technology (MIT) viscous-inviscid panel code XFOIL [33]. Unfortunately, XFOIL cannot be used for producing reliable airfoil force data after stall. For this reason, the BEM code could be used only for wind speeds not yielding rotor stall, i.e.between cut-in and rated wind speeds, although wind energy is harvested also between rated and cut-out speeds. Due to this omission, further verification of the optimal solutions presented below may be required. Such omission, however, does not hinder the demonstration of the great potential of HAWT robust design optimization, as evident in the remainder of this study.

The objective of the robust design optimization is to maximize the mean AEP and minimize its standard deviation by considering the rotor swept area corresponding to the specified rotor radius of $6.3 \mathrm{~m}$ and the site characterized by the specified Weibull wind frequency distribution, varying the 20 design variables listed above, and propagating the uncertainty affecting the 13 geometric design variables. It should be noted that in this study the rotor nominal power is an output of the optimization rather than a design specification. Mathematically, the aforementioned problem corresponds to minimize the functions $F_{1}$ and $F_{2}$ given by:

$$
\begin{aligned}
& F_{1}=-\mu_{A E P} \\
& F_{2}=\sigma_{A E P}^{2}
\end{aligned}
$$

where $\mu_{A E P}$ is the mean AEP, and $\sigma_{A E P}^{2}$ is its variance. The considered constraints are:

$$
\begin{aligned}
& C_{1}: F_{1} \leq-4 \cdot 10^{4} \quad k W h \\
& C_{2}: F_{2} \leq 2 \cdot 10^{7} \quad k W h^{2} \\
& C_{3}:\left(\mu_{B M}\right)_{\max } \leq 12 \quad k N m
\end{aligned}
$$

where $\mathrm{BM}$ denotes the blade root bending moment, and $\left(\mu_{B M}\right)_{\max }$ the maximum expectation of BM selected among the seven expectations corresponding to each considered wind speed. For each turbine, AEP is obtained by integrating the power curve against the aforementioned Weibull distribution. 
To assess the impact of considering stochastic blade geometry errors in the design optimization, a deterministic optimization, i.e.one not including uncertainty, has also been carried out. The deterministic optimization has a single-objective function, namely $F=-A E P$. The enforced constraints are:

$$
\begin{aligned}
& C_{1}: F \leq-4 \cdot 10^{4} \quad k W h \\
& C_{2}: B M_{\max } \leq 12 \quad k N m
\end{aligned}
$$

where $B M_{\max }$ is the maximum BM selected among the seven values corresponding to each considered wind speed.

\section{2 modeling aspects}

To analyze both the algorithmic and engineering aspects of the proposed probabilistic design optimization framework, the robust optimization problem presented above has been formulated and solved in several different manners. On the algorithmic side, such analyses have aimed to assess the computational cost and the robustness of the MC and URQ approaches to uncertainty propagation when using global search evolution-based optimizers. On the engineering side, two different definitions of the problem have been considered. One assumes that the shape of all three blades of a given rotor is identical, namely that identical geometry errors affect the three blades. The other assumes that such errors are different, though they are described by the same PDF. The latter scenario is more realistic, but the former has also been considered because it enables a clearer identification of the effects of stochastic geometry variability on the AEP variability. Therefore, four different robust optimizations have been performed. The first optimization (MC1) uses MC sampling and assumes that the three blades are identical; the second optimization (URQ1) uses URQ sampling, and also assumes that the three blades are identical; the third optimization (MC3) uses MC sampling and adopts a different pattern of geometry errors for the three blades of a given rotor, even though all error patterns belong to the same joint PDF; the fourth problem (URQ3) differs from MC3 only in that it uses URQ sampling for propagating uncertainty. In the MC3 case, the performance of each 'real' rotor is obtained by taking the arithmetic average of the performance of three different 'fictitious' rotors, each with identical blades affected by a different pattern of geometry errors. Conceptually, the same approach is adopted in the URQ3 case. However, the URQ method is based on a deterministic quadrature approach [11], and uses only the first four moments of the uncertainty distribution of the input variables, rather than particular instantiations of such a distribution like the MC method. Hence, the three 'fictitious' rotors of the URQ3 analysis are identical, and the performance of a rotor with blades affected by different patterns of geometry errors can be obtained by considering one rather than three rotors. Furthermore, making use of the URQ-based expressions for the expectation and the variance of output functionals [11], it is found that $a$ ) the expectation of an output using either the URQ1 or the URQ3 model for a given nominal rotor is the same, and $b$ ) the ratio of the standard deviations of an output functional computed with the URQ1 and URQ3 models is $\sqrt{3}$.

In the URQ1 and MC1 cases, a particular instantiation of the 13 geometric design variables defines the shape of the entire rotor. As the deterministic sampling of URQ requires $2 n+1$ evaluations of the functional of interest [11], with $n$ being the number of uncertain design variables, each robust analysis requires 27 computations of AEP, namely 189 WINSTRIP runs. By contrast, 10,000 AEP calculations, requiring 70,000 WINSTRIP runs are performed in the MC1 case. In the URQ3 and MC3 cases, the nominal blade geometry is still defined by 13 parameters, but, as the three blades are not identical, the number of uncertain geometry variables is $n=39$. Denoting respectively by $\mu_{1}$ and $\sigma_{1}$ the arrays of mean values and standard deviations of the geometric design variables when all three blades are identical, the mean and standard deviation arrays when 
the three blades differ are respectively $\mu_{3}=\left[\begin{array}{lll}\mu_{1} & \mu_{1} & \mu_{1}\end{array}\right]$ and $\sigma_{3}=\left[\begin{array}{lll}\sigma_{1} & \sigma_{1} & \sigma_{1}\end{array}\right]$. As mentioned above, this is because, even though in the MC3 and URQ3 cases the three blades are different, their errors are described by the same joint PDF of the URQ1 and MC1 cases. In the MC3 case, the AEP of a particular rotor is obtained by taking the arithmetic average of the AEP of three different rotors with identical blades, as explained above. Like in the MC1 problem, a set of 10,000 'real' rotors has been used to determine mean and expectation of AEP and the expectation of the BM constraint for each nominal rotor. In the MC3 problem, however, consideration of a 10,000 'real' rotors requires the generation of 30,000 blade geometries using the input PDF. In the URQ3 case, the number of AEP and constraint evaluations required for each nominal rotor is the same as for URQ1 for the reasons discussed above.

\section{Uncertainty propagation}

Here the URQ and MC sampling techniques are assessed and compared both in terms of accuracy and computational costs and accuracy. Extensive numerical testing has shown that the use of a 10,000-rotor set with both MC1 and MC3 sampling yields fully converged estimates of both the expectation and the standard deviation of AEP and BM. For this reason, this number of samples has been maintained throughout the MC1 and MC3 optimization problems. As discussed in the preceding section, the number of rotor configurations to be analyzed to obtain the mean and the expectation of AEP and BM. This highlights the substantial computational cost achieved by using URQ rather than MC sampling.

As shown in the next section, the considered type of optimization problem leads to a Pareto front arising from the trade-off between the expectation and the standard deviation of AEP. In the following, the MC and URQ estimates of the mean and standard deviations of the output functionals are cross-compared for two nominal rotors: a non-optimal rotor, namely one lying relatively far from the Pareto front, and an optimal rotor lying on the Pareto front. The comparison of the MC and URQ expectations and standard deviations of AEP and BM of the non-optimal nominal rotor is presented in Table 2 . The symbol $\left(\mu_{B M}\right)_{\max }$ denotes the maximum expectation of BM selected among the seven expectations corresponding to each considered wind speed, whereas the symbol $\left(\sigma_{B M}\right)_{\max }$ is the standard deviation of BM at the wind speed associated with $\left(\mu_{B M}\right)_{\max }$. These results highlight that the expectation of both functionals does not depend on whether the blades of a given rotor are taken to be identical or not. An excellent agreement between the MC and URQ expectations is also observed. Two main observations emerges from the inspection of the standard deviations of Table 2. Firstly, for a given propagation method, the estimate of $\sigma_{A E P}$ obtained by taking all three blades to be identical is higher than that obtained by assuming that the three blades differ. The URQ approach predicts the ratio between the former and the latter standard deviations to be $\sqrt{3}$. Secondly, the difference between the MC1 and URQ1 estimates of $\sigma_{A E P}$ is about $5.7 \%$, whereas that between the MC3 and the URQ3 estimates of $\sigma_{A E P}$ is about $2.9 \%$.

To further investigate the differences between the URQ and MC estimates of expectation and standard deviation, the analysis of an optimal nominal rotor is now considered. The MC and URQ expectations and standard deviations of AEP and BM of the optimal nominal rotor are reported in Table 3. These results confirm that the expectation of both functionals does not depend on whether the three rotor blades are taken to be identical or not. An excellent agreement between the MC and URQ expectations is observed also for this rotor. Also in this case, the estimates of $\sigma_{A E P}$ obtained by considering all three blades to be identical is higher than that obtained by assuming that different geometry errors affect the three rotor blades. The agreement between the MC and the 
URQ predictions of $\sigma_{A E P}$ is slightly worse than for the non-optimal rotor: the difference between the MC1 and URQ1 values of $\sigma_{A E P}$ is about $9.0 \%$, and that between the MC3 and the URQ3 estimates is about $11.0 \%$.

\begin{tabular}{|c|c|c|c|c|}
\hline & $\mu_{A E P}$ & $\left(\mu_{B M}\right)_{\max }$ & $\sigma_{A E P}$ & $\left(\sigma_{B M}\right)_{\max }$ \\
\hline URQ1 & 42150.4 & 5.2136 & 2432.1 & 0.5719 \\
\hline MC1 & 42126.9 & 5.2051 & 2580.1 & 0.5742 \\
\hline URQ3 & 42150.4 & 5.2136 & 1404.2 & 0.5719 \\
\hline MC3 & 42123.7 & 5.2016 & 1445.9 & 0.5762 \\
\hline
\end{tabular}

Table 2: Expectations and standard deviations of AEP $[k W h]$ and BM $[k N m]$ of a non-optimal nominal rotor based on MC and URQ sampling.

\begin{tabular}{|c|c|c|c|c|}
\hline & $\mu_{A E P}$ & $\left(\mu_{B M}\right)_{\max }$ & $\sigma_{A E P}$ & $\left(\sigma_{B M}\right)_{\max }$ \\
\hline URQ1 & 91619.2 & 11.9999 & 2778.8 & 1.3593 \\
\hline MC1 & 91745.8 & 12.0136 & 3056.4 & 1.3504 \\
\hline URQ3 & 91619.2 & 11.9999 & 1604.4 & 1.3593 \\
\hline MC3 & 91760.6 & 12.0376 & 1784.4 & 1.3545 \\
\hline
\end{tabular}

Table 3: Expectations and standard deviations of AEP $[k W h]$ and BM $[k N m]$ of an optimal nominal rotor based on MC and URQ sampling.

In absolute terms, the differences between the URQ and the reference MC estimates of $\sigma_{A E P}$ observed over the considered design space appear to be relatively high. In general, the accuracy of URQ is fairly problem-dependent, and is expected to decrease when the output functionals feature a strong nonlinear dependence on the input variables. Such a reduction also increases with the size of the standard deviation of the input variables. The reported differences between the MC and URQ estimates of $\sigma_{A E P}$ point to the existence of significant nonlinearities of the BEM-based analysis. In other robust optimizations making use of URQ for propagating uncertainty [9, 34], the discrepancies between the URQ and MC estimates of the standard deviations of the output functionals has been found to be substantially smaller than in the present case, presumably due to smaller nonlinearities of the output functionals. The level of nonlinearity of the BEM-based analysis can be assessed by examining the AEP PDF determined with MC sampling. The histograms of the left and right subplots of Fig. 2 depict the MC1-based AEP PDF of the non-optimal and optimal nominal rotors respectively. In both subplots, the curve labeled 'gaussian' is the normal distribution with mean and standard deviation determined by MC1 sampling, whereas the curve labeled 'parzen' is the continuous counterpart of the MC1 histogram. The deviation of the parzen fit from the gaussian curve can be taken as a measure of the nonlinearity of the BEM-based analysis. This is because a linear function would map a normally distributed input onto a normally distributed output. Inspection of the results reported in Fig. 2 reveals that both the BEM analysis centered at the nonoptimal nominal rotor configuration and that centered at the optimal rotor configuration present a significant degree of nonlinearity, and also that the nonlinearity is higher in the case of the latter rotor configuration. These findings account for the observed discrepancies between the URQ- and MC-based estimates of $\sigma_{A E P}$.

Despite these deviations of the URQ estimates of the standard deviations from the reference $\mathrm{MC}$ results, the use of URQ for the robust design optimization problems considered herein is 
believed to be appropriate. This is because, as shown in the next section, the reductions of $\sigma_{A E P}$ obtained by performing a robust design optimization, based on either URQ or MC uncertainty propagation, rather than a deterministic design optimization are substantially higher than the observed discrepancies between the URQ and MC estimates of $\sigma_{A E P}$. Furthermore, a significant advantage of the URQ uncertainty propagation technique is its substantially lower computational cost with respect to the MC uncertainty propagation.
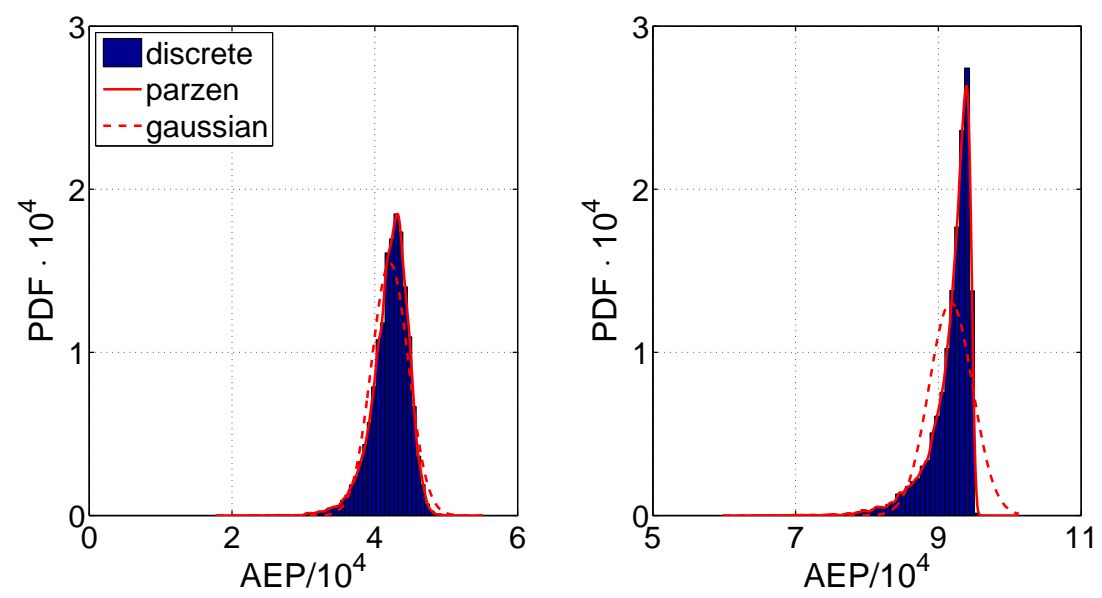

Figure 2: Analysis of AEP [kWh] PDFs. Left subplot: non-optimal nominal rotor. Right subplot: optimal nominal rotor.

\section{Results}

\section{1 robust optimizations}

The adopted formulation of the robust design optimization problem leads to a Pareto front arising from the trade-off between the expectation and the standard deviation of AEP. The URQ1 and MC1 Pareto fronts obtained with MOPED are reported in the left subplot of Fig. 3. The figure also reports the probabilistic performance of three additional rotors. One, the configuration labeled 'Turbine A', is the non-optimal rotor considered for the comparative analysis of the URQ and MC methods reported in the preceding section. The other two configurations, labeled 'URQ1 ref.' and 'MC1 ref.' correspond to the nominal rotor geometries obtained by using the IDEA local refinement starting from a point with maximum $\mu_{A E P}$ taken from the URQ1 and MC1 Pareto front respectively. In both cases, the refinement is performed as follows: given the final population of the MOPED optimization, a sub-population containing a solution with maximum $\mu_{A E P}$ is selected and used as starting point of the IDEA optimization. This optimization aims at maximizing $\mu_{A E P}$ subject to the constraints (4). The solution labeled 'URQ1 ref.' is the optimal rotor considered in URQ/MC cross-comparison in the preceding section. The results provided by the left subplot of Fig. 3 show that a wide range of Pareto-optimal values of $\mu_{A E P}$ exists, and that a substantially smaller range of $\sigma_{A E P}$ corresponds to this $\mu_{A E P}$ range. Even rotors featuring high values of $\mu_{A E P}$ present relatively low values of $\sigma_{A E P}$.

The URQ3 and MC3 Pareto fronts of $\mu_{A E P}$ and $\sigma_{A E P}$ obtained with MOPED are reported in the right subplot of Fig. 3. The subplot also reports the probabilistic performance of two additional rotors. These two configurations, labeled 'URQ3 ref.' and 'MC3 ref.' have been obtained by using 
the IDEA local refinement in the same manner as described above the URQ1 and MC1 problems. One notes that also in the MC3 and URQ3 cases a wide range of Pareto-optimal values of $\mu_{A E P}$ exists, and that a substantially smaller range of $\sigma_{A E P}$ corresponds to this $\mu_{A E P}$ range. Furthermore, the values of the Pareto-optimal values of $\sigma_{A E P}$ are slightly lower than in the URQ1 and MC1 cases, as expected.

On the basis of the URQ expressions for mean and standard deviation of an output functional [11], a given nominal rotor has the same $\mu_{A E P}$ in the URQ1 and URQ3 cases. The URQ1 and URQ3 estimates of $\sigma_{A E P}$ are instead different, and their ratio is $\sqrt{3}$. Due to the adopted definition of the BM constraints, the URQ1 and URQ3 estimates of both $\left(\mu_{B M}\right)_{\max }$ and $\left(\sigma_{B M}\right)_{\max }$ are equal. Consequently, if none of the constraints depending on the geometry errors of the rotor blades were active at the end of the optimization, and the optimizer converged to the best possible approximation of the true Pareto front, the URQ1 and URQ3 Pareto fronts would correspond to the same set of rotors. This is because, in the two two-objective optimizations, one objective function $\left(\mu_{A E P}\right)$ is the same, and the other $\sigma_{A E P}$ differs by a constant scaling factor. In line with these considerations, the computed URQ1 and URQ3 Pareto fronts have found to differ only for a constant scaling factor of $\sigma_{A E P}$. Moreover, it has been found that the rotors corresponding to the optimal configurations labeled 'URQ1 ref.' in the left subplot of Fig. 3 and 'URQ3 ref.' in the right subplot of Fig. 3 present very small differences.
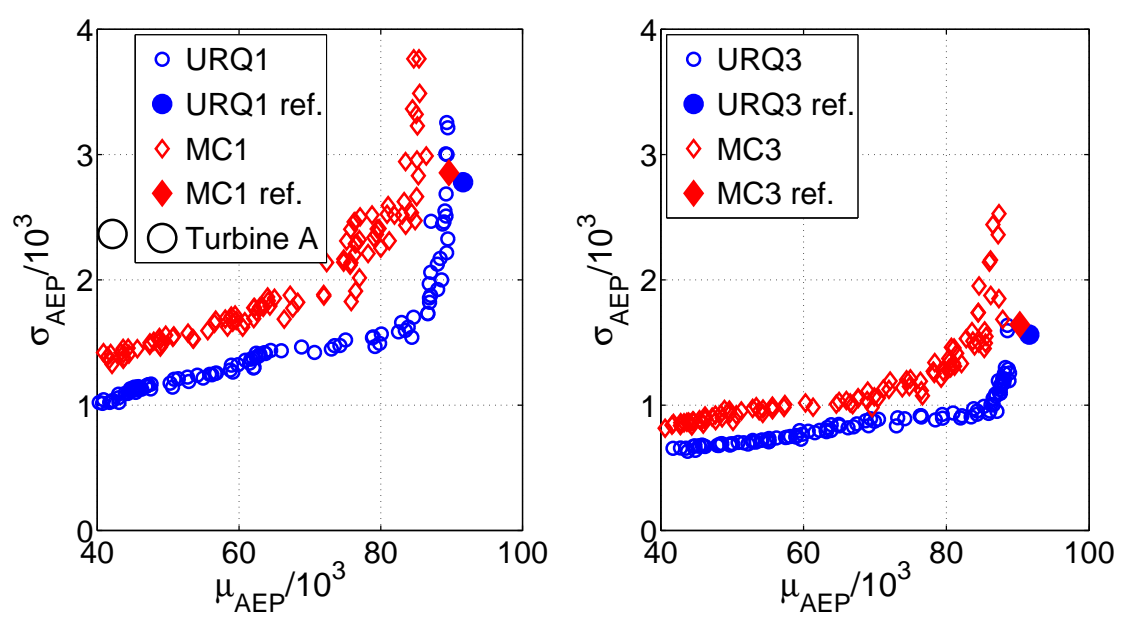

Figure 3: Pareto fronts of expectation and standard deviation of AEP [kWh]. Right subplot: URQ1 and MC1 fronts. Left subplot: URQ3 and MC3 fronts.

The observed differences between the URQ1 and MC1 Pareto front estimates, and those between the URQ3 and MC3 Pareto front estimates highlight that the choice of the uncertainty propagation technique has some influence on the solution of the robust optimization problem. The main reason for such differences is a different converge rate of the MOPED optimizer using MC or URQ uncertainty propagation. The MC and URQ approaches have different sensitivity to the 'irregularities' of the design space: when the neighborhood of a nominal rotor contains one or more rotors for which WINSTRIP does not converge, the MC approach is more likely than the URQ approach to sample one or more of these 'defective' solutions; in this circumstance, since an undetermined solution cannot be used to compute the statistical characteristics of the performance, the nominal geometry itself is considered defective and is discarded. When the URQ propagation is used, MOPED is able to converge more rapidly in regions of the search space corresponding to higher amounts of energy production, while the high level of noise of the MC sampling slows down 
or even prevents full convergence to the Pareto front. Thus, the use of MC sampling forces the optimizer to act in a conservative fashion, due to the excessive sensitivity of this sampling strategy to irregularities of the design space. Such irregularities, however, do not necessarily correspond to actual characteristics of the real system. Indeed, the fact that the BEM analysis of certain rotor geometries fails, does not necessarily imply that such configurations would deliver no power at all. To a minor extent, an additional possible contribution to the observed differences between the URQ and MC Pareto fronts could be the difference observed in some regions of the design space between the standard deviation of the output functionals determined with the URQ and MC sampling. This factor, however, is not believed to have a strong weight, since the differences between the URQ and $\mathrm{MC}$ estimates of $\sigma_{A E P}$ of Figure 3 are larger than the maximum differences between the URQ and MC estimates of $\sigma_{A E P}$ associated with a given nominal rotor.

One of the reasons why evolution-based rather than gradient-based optimization has been used in this study is the existence of the aforementioned irregularities of the design space. Gradient-based optimization can also cope with the occurrence of infeasible areas during the search, for example by dynamically varying size and direction of the search step. The occurrence of an infeasible point in evolution-based optimization, however, is less perturbing, since the optimizer continues its search and simply ignores infeasible designs. The convergence to global optima could also be severely slowed down or even prevented by the existence of local minima in a fairly wide design space like that considered in this study. Furthermore, the use of MC-like sampling to compute robust functionals is typically affected by numerical noise, and this feature would also create additional difficulties to the convergence of gradient-based optimization. These are the main reasons why evolution-based optimization has been selected for this study.

Both the URQ and MC optimizations use 100 generations with 100 individuals each. This require 10,000 probabilistic function evaluations. Each probabilistic evaluation, however, requires consideration of 10,000 rotors (or blades) in the MC cases, and only 27 rotors in the URQ cases. thus, the ratio between the overall computational cost of the MC1 and URQ1 optimizations is about 370. In the MC3 case, each probabilistic function evaluation requires consideration of 30,000 rotors with identical blades. Hence, the ratio between the overall computational cost of the MC3 and URQ3 optimizations is about 1,110.

The fitness parameter $\alpha_{f}$ and the sampling proportion $\tau_{E}$ for all MOPED-based optimizations have been set to 0.5 and 1 , respectively. In all IDEA-based optimizations, the weighting factor $F$ and the crossover probability $C R$ have both been set to 0.9 . The IDEA search has used a random population of 20 individuals, and has stopped when the population has contracted to $25 \%$ of the maximum expansion during the evolution.

\section{2 robust and deterministic optima}

The optimal rotor obtained by solving the deterministic optimization problem described in section 3 has a nominal AEP of 96,170 $\mathrm{kWh}$, and $\mu_{A E P}=89,974 \mathrm{kWh}$. Here, this rotor configuration, denoted by 'det. opt.', is compared to the 'URQ1 ref.' rotor, which has a nominal $A E P$ of 95,005 $\mathrm{kWh}$ and $\mu_{A E P}=91,619 \mathrm{kWh}$. The AEP standard deviation of the 'det. opt.' rotor is $\sigma_{A E P}=4,991 \mathrm{kWh}$, and is significantly higher than that of the 'URQ1 ref.' rotor, which has $\sigma_{A E P}=2,779 \mathrm{kWh}$. These figures highlight that the AEP standard deviation of the rotor designed taking into account stochastic geometry errors due to finite manufacturing and assembly tolerances is more than $44 \%$ lower than that of the rotor designed neglecting such errors.

The performance of these two rotors is compared in greater detail in the three subplots of Fig. 4. The left subplot compares power curves, the middle subplot compares the amount of AEP accounted for by each wind speed $U$, and the right subplot compares the blade root bending 
moment against $U$. In all three subplots, the labels 'nominal' and 'mean' denote respectively deterministic values computed using the nominal rotor geometries and expected values taking into account stochastic geometric uncertainties. All mean curves also report error bars below and above the ordinate of each curve base-point, and the size of each bar is the standard deviation of the considered output. Examination of the left and middle subplots reveals that the 'det. opt.' rotor has a slightly better nominal aerodynamic performance, but slightly worse mean performance than the 'URQ1 ref.' rotor. More importantly, however, the standard deviations of both power and energy production of the deterministically optimal rotor are significantly higher than those of the probabilistically optimal rotor. This confirms that the use of robust design optimization yields HAWT rotors which outperform, in a statistical sense, those obtained by using the deterministic design approach, which neglects probabilistic geometry errors. The right subplot of Fig. 4 highlights that the standard deviation of the bending moment of the probabilistically optimum rotor is lower than that of the deterministically optimum rotor for all considered wind speeds. The mean and the nominal values of this output for the two rotors are similar for all considered wind speeds, except for the speed of $12 \mathrm{~m} / \mathrm{s}$, there the mean value of the probabilistic solution is slightly higher than that of the deterministic solution. This may result in higher fatigue loads on the probabilistically designed rotor. This problem could be solved by including a fatigue load-related constraint in the probabilistic design optimization system.
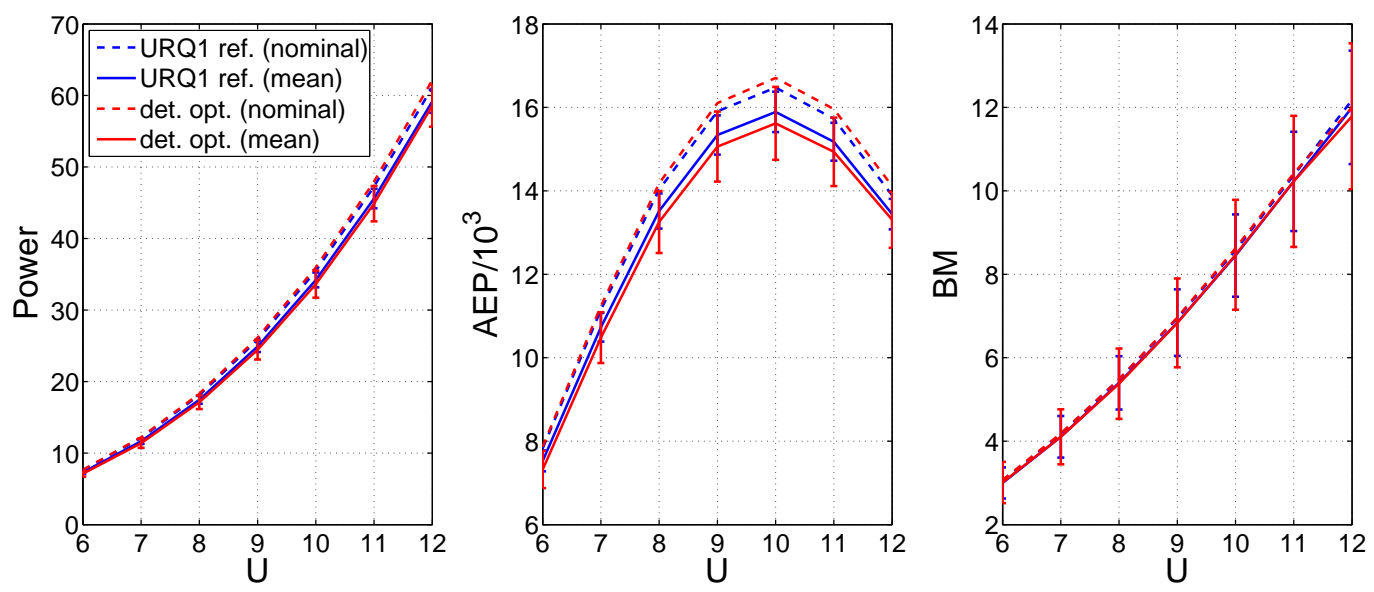

Figure 4: Rotor performance of deterministic and robust designs. Left subplot: mechanical power $[k W]$ against wind speed $U[m / s]$. Middle subplot: proportion of AEP $[k W h]$ accounted for by each wind speed. Right subplot: blade root bending moment $[k N m]$ against wind speed.

The radial profiles of the pitch angle $\theta_{p}$ and the chord $c$ of the two rotors are reported respectively in the top left and bottom left subplots of Fig. 5. The red curve of these two subplots is the percentage variation $(\Delta \%)$ of these variables. One sees that the sectional pitch angle of the probabilistically optimal rotor blade is smaller than that of the deterministically optimal blade over most part of the blade length, featuring a maximum reduction of about $16 \%$ at $4 \mathrm{~m}$ blade length. The chord of the probabilistic optimum is wider than that of the deterministic optimum over the entire blade length, featuring a maximum increment of nearly $60 \%$ at the blade tip. The pitch angle of the probabilistically optimal rotor and, to a minor extent, that of the deterministically optimal rotor, are negative over part of the blade height. Fixed-pitch HAWTs, like those considered in this study, rely on the aerodynamic torque of the hub region of the blades for starting from a standstill [35]. One of the prerequisites for achieving a sufficiently high start-up torque is that the 
effective angle of attack in the hub region be not too high. If the local pitch angle is not too low, the start-up torque may be increased by decambering the airfoils of the hub region, Modern wind turbines of this size, however, can also be non-self-starting and started by external means. In general, if a self-starting machine is required, the constraint of given minimum positive aerodynamic torque at start-up could also be included in the formulation of the design optimization problem, though such a constraint has not been adopted in the presented optimizations. The rotational speed of the two rotors for all considered wind speeds is reported in the right subplot of Fig. 5, which highlights that the probabilistically optimal rotor has lower $\Omega$ values than the deterministically optimal rotor. The slope discontinuities of the $\Omega$-curves at $U=11 \mathrm{~m} / \mathrm{s}$ occur because the BM constraint becomes active at $U=12 \mathrm{~m} / \mathrm{s}$, as visible in the right subplot of Fig. 4, The fulfillment of the BM constraint is achieved through a reduction of the aerodynamic forces acting on the blade, and such a reduction is achieved by slightly reducing the rotational speed with respect to the value it would have taken if the structural constraint had remained inactive. This occurrence highlights that the selected type and level of structural constraint are overly restrictive, since the rotational speed is usually not limited by structural requirements before the rated wind speed. More realistic design specifications would consider this type of structural constraint applied to extreme load conditions. This is an item for future extensions of this work.

The left subplot of Fig. 4 shows that the power curves of the two optimal rotors differ fairly little from each other. This is because the effects of the variations of the geometric and operational parameters on the turbine power output balance out. At a wind speed of $12 \mathrm{~m} / \mathrm{s}$, for example, the reduction of the square of the rotational speed of the robust optimum relative to the deterministic optimum is about $27 \%$, which constitutes a negative contribution to the power of the robust optimum. This reduction, however, is balanced by the power increment associated with the wider chords and the smaller pitch angles of the probabilistic optimum. This is highlighted by the radial profiles of the percentage variations of $\theta_{p}$ and $c$ reported respectively in the top left and bottom left subplots of Fig. 5. As visible in these two subplots, the variation of these two geometric parameters is of the same order of magnitude as that of the square of the rotational speed. It is also observed that the extreme aerodynamic loads on the blades of the probabilistically designed rotor may be higher than those on the blades of the deterministically optimized rotor due to the wider chord of the former configuration. This aspect can be easily accounted for by including extreme load constraints in the design frameworks presented herein.

To further verify the correctness of the comparative analysis of the two designs, the nominal and stochastic performance of the 'det. opt.' rotor geometry using the rotational speeds of the 'URQ1 ref.' rotor have been computed. The nominal and mean AEP of this hybrid configuration are $A E P=91,191 \mathrm{kWh}$ and $\mu_{A E P}=88,092 \mathrm{kWh}$ respectively. More importantly, the hybrid rotor has $\sigma_{A E P}=5,197 \mathrm{kWh}$, which is significantly higher than $\sigma_{A E P}$ of the deterministic design 'det. opt.'. Thus, the performance of the hybrid configuration is poorer than that of both the deterministic and probabilistic optima, and this confirms the self-consistency of the deterministic and probabilistic optimizations.

The analyses above indicate that a lower sensitivity of AEP to rotor geometry errors can be achieved by lowering rotational speeds and compensating for the reduction of power due to lower rotational velocities by shifting upwards the radial profiles of $C_{L}$. This increment of the aerodynamic loading is achieved by increasing the angle of attack (to a large extent through lower values of the sectional pitch) and the chord of the blade over most part of the blade length. This is the reason why the 'URQ1 ref.' blade has lower values of $\theta_{p}$ : lower values of the section pitch angle lead to higher values of $\alpha$. An additional contribution to the increment of $\alpha$ comes from the reduced circumferential speed, which results in higher values of the relative wind angle $\phi$. The radial profiles of the mean value of $\alpha$ of the two rotors at $U=12 \mathrm{~m} / \mathrm{s}$ are reported in the left subplot of Fig. 6 , 
which confirms that the angle of attack along the 'URQ1 ref.' blade is higher than that of the 'det. opt.' blade. Note also that the standard deviation of $\alpha$ at a given radial position is about the same for the two rotors. This is due to the fact that equal errors of the section pitch angle lead to equal variations of $\alpha$. The higher values of the mean radial profile of $\alpha$ of the 'URQ1 ref.' blade result in significantly higher mean values of $C_{L}$, as shown in the right subplot of Fig. 6. It is important to note that the standard deviation of $C_{L}$ of the blade 'det. opt.' is between about 10 and $40 \%$ higher than that of the blade 'URQ1 ref.' over most part of the blade. Such lower values occur because the overall level of $\alpha$ is in a region where the slope of the $\alpha-C_{L}$ curve starts to decrease with respect to the linear part corresponding to lower angles of attack. Therefore, the variation of the lift coefficient caused by a given variation of $\alpha$ is smaller for the 'URQ1 ref.' blade. The lower values of the standard deviation of $C_{L}$ are the main reason for the lower standard deviation of the annual energy production of the probabilistically optimized rotor with respect to that of the deterministically optimized rotor.
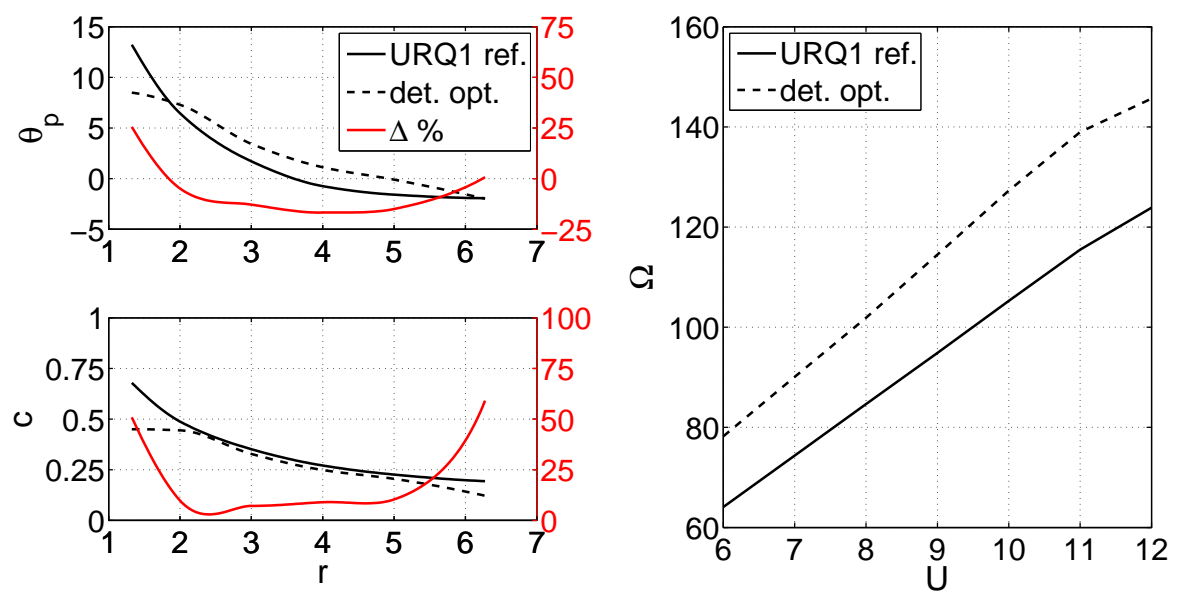

Figure 5: Rotor geometry and control of deterministic and robust designs. Top left subplot: blade chord $c[m]$ against radius $r[m]$. Bottom left subplot: section pitch angle $\theta_{p}[d e g]$ against radius. Right subplot: rotational speed $\Omega[R P M]$ against wind speed $U[\mathrm{~m} / \mathrm{s}]$.

\section{Conclusions}

A novel robust optimization framework for the design of variable-speed HAWT rotors has been presented. The effectiveness of the developed technology has been demonstrated by considering the design optimization of a three-blade rotor with blades affected by randomly distributed geometry errors caused by manufacturing and assembly tolerances. The developed technology has been used to maximize the expectation of the annual energy production and minimize its standard deviation. This multi-objective optimization problem has been solved with an effective two-stage multi-objective evolution-based optimization strategy.

Two alternative methods for propagating uncertainty through the design chain have been considered: a standard Monte Carlo approach and the Univariate Reduced Quadrature. The level of agreement between the Monte Carlo and URQ estimates of mean and standard deviations of the output functionals of interest has been found to be adequate for the considered robust design applications. The latter approach reduces the cost of each robust analysis by up to three orders of magnitude with respect to the case in which standard Monte Carlo sampling is adopted. 

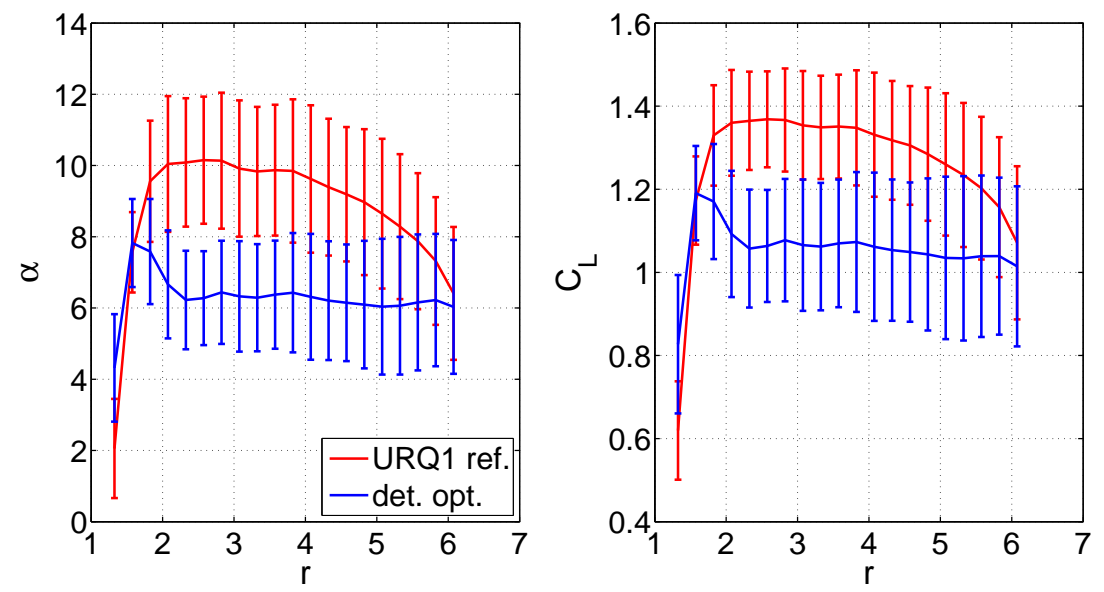

Figure 6: Rotor aerodynamics of deterministic and robust designs at $U=12 \mathrm{~m} / \mathrm{s}$. Left subplot: angle of attack $\alpha[\mathrm{deg}]$ against radius $r[\mathrm{~m}]$. Right subplot: lift coefficient $C_{L}$ against radius.

The comparative analysis of a rotor design obtained by considering the stochastic geometry errors, and a rotor design obtained by neglecting all uncertainties shows that the AEP standard deviation of the former rotor is less than $40 \%$ that of the latter. The feasibility of the particular probabilistic stall-regulated rotor design presented herein needs to be further assessed from several other viewpoints, including the choice of the rotor airfoils and the inclusion of the stalled rotor regime (portion of the power curve from rated to cut-out wind speed) in the calculation of AEP. These verifications were beyond the scope of the paper, and are ground for extensions of this work. This was a prototype study, and one of its main objectives was to explore the potential of robust design optimization for general HAWT rotor probabilistic design. This approach hopefully offers a new way of incorporating several uncertainty sources in general HAWT design.

\section{Acknowledgements}

This project was carried out with support of the wind turbine manufacturer Gaia-Wind, which is hereby acknowledged. Ms. Sara Myers is kindly acknowledged for editing this paper.

\section{References}

[1] P. Fuglsang and H.A. Madsen. Optimization method for wind turbine rotors. Journal of Wind Engineering and Industrial Aerodynamics, 80(1):191-206, 1999.

[2] P. Fuglsang, C. Bak, J.G. Schepers, B. Bulder, T.T. Cockerill, P. Claiden, A. Olesen, and R. van Rossen. Site-specific Design Optimization of Wind Turbines. Wind Energy, 5(4):261$279,2002$.

[3] W. Xudong, W.Z. Shen, W.J. Zhu, J.N. Sørensen, and C. Jin. Shape Optimization of Wind Turbine Blades. Wind Energy, 12(8):781-803, 2009.

[4] M. Doessing, H.A. Madsen, and C. Bak. Aerodynamic optimization of wind turbine rotors using a blade element momentum method with corrections for wake rotation and expansion. Wind Energy, 15(1):563-574, 2012. 
[5] E. Benini and A. Toffolo. Optimal design of horizontal-axis wind turbines using blade-element theory and evolutionary computation. Journal of Solar Energy Engineering, 124:357-363, 2002 .

[6] J.C. Helton and F.J. Davis. Latin hypercube sampling and the propagation of uncertainty in analyses of complex systems. Reliability Eng. System Safety, 81(1):23-69, 2003.

[7] J.C. Helton and F.J. Davis. Efficient uncertainty analysis methods for multidisciplinary robust design. AIAA Journal, 40(3):545-552, 2002.

[8] M. Putko, P. Newman, A. Taylor, and L. Green. Approach for uncertainty propagation and robust design in CFD using sensitivity derivatives. In Proceedings of the 15th AIAA Computational Fluid Dynamics Conference, pages 1-10, Anaheim CA, 2001. AIAA-20012528.

[9] M. Padulo, M.S. Campobasso, and M.D. Guenov. Comparative analysis of uncertainty propagation methods for robust engineering design. In International Conference on Engineering Design ICED0\%, pages 1-12, Paris, France, 2001.

[10] D. Xiu and E.M. Karniadakis. Modeling uncertainty in flow simulations via generalized polynomial chaos. Journal of Computational Physics, 187:137-167, 2003.

[11] M. Padulo, M.S. Campobasso, and M.D. Guenov. A novel uncertainty propagation method for robust aerodynamic design. AIAA Journal, 49(3):530-543, 2011.

[12] D. Wood. Small Wind Turbines. Springer, New York, USA, 2011.

[13] T. Kramkowski, D. Foussekis, T. Chaviaropoulos, and G. Ronsten. Harmonisation and improvement of rotor blade quality control. Contract Report Jor3-CT95-0092, Deutsches Windenergie-Institut gem. GmbH (DEWI), Wilhelmshaven, Germany, 1997. Available from: http://cordis.europa.eu/documents/documentlibrary/47698161EN6.pdf (Accessed 1 October 2014).

[14] G. Petrone, C. de Nicola, D. Quagliarella, J. Witteveen, and G. Iaccarino. Wind Turbine Performance Analysis Under Uncertainty. In Proceeding of the 49th AIAA Aerospace Sciences Meeting including the New Horizons Forum and Aerospace Exposition, pages 1-18, Orlando FL, January 2011. AIAA-2011544.

[15] G. Petrone, C. de Nicola, D. Quagliarella, J. Witteveen, J. Axerio-Cilies, and G. Iaccarino. Wind Turbine Optimization Under Uncertainty with High Performance Computing. In Proceeding of the 29th AIAA Applied Aerodynamics Conference, pages 1-16, Honolulu HI, June 2011. AIAA-20113806.

[16] M. Costa and E. Minisci. MOPED: a multi-objective parzen-based estimation of distribution algorithm. In EMO 2003, pages 282-294, Faro, Portugal, 2003. Springer.

[17] G. Avanzini, D. Biamonti, and E. Minisci. Minimum-fuel/minimum-time maneuvers of formation flying satellites. In Adv. Astronaut. Sci., volume 116, pages 2403-2422, 2003.

[18] M. Vasile, E. Minisci, and M. Locatelli. An inflationary differential evolution algorithm for space trajectory optimization. Evolutionary Computation, IEEE Transactions on, 15(2):267$281,2011$. 
[19] G. Pechlivanoglou, G. Weinzierl, I.T. Masmanidis, C.N. Nayeri, T.P. Philippidis, and C.O. Paschereit. Utilization of Modern Large Scale HAWT Blade Design Technologies for the Development of Small HAWT Blades. In ASME Turbo EXPO Technical Conference, pages 1-9, Düsseldorf, Germany, June 2014. GT2014-25309.

[20] J.A. Lozano, P. Larranaga, I. Inza, and E. Bengoetxea. Towards a New Evolutionary Computation: Advances on Estimation of Distribution Algorithms (Studies in Fuzziness and Soft Computing). Springer, New York, USA, February 2006.

[21] K. Fukunaga. Introduction to statistical pattern recognition. Academic Press, Waltham, USA, 1972.

[22] K. Deb, A. Pratap, S. Agarwal, and T. Meyarivan. A Fast and Elitist Multiobjective Genetic Algorithm: NSGA-II. Evolutionary Computation, IEEE Transactions on, 6(2):182-197, 2002.

[23] D. Datta, K. Deb, C.M. Fonseca, F.G. Lobo, P.A. Condado, and J. Seixas. Multi-objective evolutionary algorithm for land-use management problem. International Journal of Computational Intelligence Research, 3(4):371-384, 2007.

[24] K. Deb. Scope of stationary multi-objective evolutionary optimization: A case study on a hydro-thermal power dispatch problem. Journal of Global Optimization, 41(4):479-515, 2008.

[25] R.H. Leary. Global optimization on funneling landscapes. Journal of Global Optimization, 18(4):367-383, 2000.

[26] K. Price, R.M. Storn, and J.A. Lampinen. Differential Evolution: A Practical Approach to Global Optimization. Springer, New York, USA, 2005.

[27] J.F. Manwell, J.G. McGowan, and A.L. Rogers. Wind Energy Explained, Second Edition. Wiley, Hoboken, USA, 2009.

[28] J. Manwell, J. McGowan, and A. Rogers. Wind Energy Explained. Theory, Design and Application. John Wiley and Sons Ltd., Hoboken, USA, 2002.

[29] M. Buhl. A new empirical relationship between thrust coefficient and induction factor for the turbulent windmill state, August 2005. http://www.nrel.gov/docs/fy05osti/36834.pdf.

[30] H. Snel, R. Houwink, and J. Bosschers. Sectional prediction of lift coefficients on rotating wind turbine blades in stall, 1994.

[31] D. Spera. Models of lift and drag coefficients of stalled and unstalled airfoils in wind turbines and wind tunnels, 2008.

[32] M.M. Hand, D.A. Simms, L.J. Fingersh, D.W. Jager, J.R. Cotrell, S. Schreck, and S.M. Larwood. Unsteady aerodynamics experiment phase VI: Wind tunnel test configurations and available data campaigns. Technical report, National Renewable Energy Laboratory (NREL), Golden, USA, 2001. pages 1-299.

[33] M. Drela. XFOIL: An Analysis and Design System for Low Reynolds Number Airfoils. In Low Reynolds Number Aerodynamics, volume 54 of Lecture Notes in Engineering, pages 1-12, New York, USA, 1989. Springer-Verlag.

[34] M. Padulo, B. Lee, and M. Liou. Probabilistic design of flapping airfoils in gusty environments. AIAA-2011-2036, 2011. 
[35] P. Brøndsted and R.P.L. Nijssen editors. Advances in wind turbine blade design and materials. Woodhead Publishing Limited, Sawston, UK, 2013. 